\title{
REGOLAMENTAZIONE, REDDITIVITÀ E FUTURO DELLE BANCHE
}

\author{
ROBERTO RUOZI (*)
}

SuNTO. - L'eccesso di regole alle quali è sempre più sottoposta l'attività bancaria, l'avversione al rischio - condizionata anche dalla paura di ripetere errori che hanno così pesantemente afflitto i bilanci e le performance borsistiche - e il ruolo cruciale assunto dal patrimonio nelle banche sono i principali fenomeni che mantengono la redditività delle banche stesse a livelli molto bassi. Il problema è importantissimo per il futuro delle banche. Innanzitutto, se la redditività non migliora le possibilità di aumentare il patrimonio per perseguire obiettivi di sviluppo dimensionale sono nulle. Non ci sarà infatti autofinanziamento e non sarà possibile reperire nuovo capitale nel mercato, in cui gli investitori troveranno opportunità migliori. Nei limiti in cui il patrimonio continuerà ad essere al centro dell'attenzione dei regolatori e dei controlli di vigilanza, questo significherà il blocco delle banche e la riduzione della loro propensione alla concessione dei crediti.

ABSTRACT. - The excess of regulation that bank activities are increasingly subject to, the risk aversion that is also conditioned by the fear of repeating the mistakes that have heavily plagued the economic and stock market performances, and the crucial role of bank capital are the main factors that maintain the profitability of the banks at very low levels. This is a considerable problem for the future of banks. First, if profitability does not improve, the possibility of increasing capital to pursue growth and development are zero. There will be no self-financing and it will not be possible to raise new capital in the market in which investors will find better opportunities. To the extent that capital will continue to be the focus of regulatory and supervisory controls, this will imply the blockage of the banks and the reduction of their propensity to give credit to the economy.

La crisi iniziata nel 2007 e non ancora del tutto terminata ha provocato in tutto il mondo, seppure con intensità e modalità diverse,

(*) Istituto Lombardo Accademia di Scienze e Lettere, Milano; Università Commerciale Luigi Bocconi, Milano, Italia. E-mail: apbsrl@gmail.com 
profondi mutamenti nell'attività bancaria e nella gestione delle banche. Molti di questi mutamenti sono in effetti già avvenuti, mentre altri sono ancora in corso e altri saranno prodotti in futuro.

I governi e le autorità nazionali e internazionali di vigilanza hanno reagito alla suddetta crisi in modo vario. In un primo momento gli interventi sono stati più che altro volti ad impedire il fallimento delle banche e, in particolare, di quelle con valenze e rischi sistemici. I salvataggi effettuati dagli Stati hanno assunto dimensioni colossali (si parla di circa 6.000 miliardi di dollari a livello globale) ed hanno enormemente gravato sui bilanci statali sollevando forti polemiche sulla loro «equità». Il dibattito ha riguardato essenzialmente il giudizio sul fatto di aver messo a carico dei contribuenti il peso dei danni provocati da manager bancari superpagati, corrotti, incapaci o anche semplicemente sfortunati.

In un secondo momento si è entrati nell'analisi delle cause della crisi, che in estrema sintesi possono essere riassunte nell'eccessiva propensione al rischio mostrata dalle banche, che in effetti ad un certo momento non sono più state in grado di gestire i rischi assunti e si sono avviate verso l'insolvenza.

In un terzo momento l'attenzione delle autorità si è spostata sulle misure da adottare per evitare nuove crisi e nel caso in cui, nonostante le misure adottate, esse si ripetessero si è dedicata particolare cura nell'impostare le procedure della loro gestione in modo da non penalizzare i contribuenti, scaricandone il costo soprattutto sugli azionisti e sui creditori delle banche in crisi.

La prima serie di provvedimenti si è concentrata sull'impostazione di regole per ridurre i rischi dell'attività bancaria, collegandoli strettamente ad alcune variabili chiave della gestione, come il patrimonio e la liquidità. Si sono anche imposte regole che limitano o vietano determinate attività considerate eccessivamente rischiose e peraltro poco utili allo sviluppo dell'economia. Inoltre, grandi sforzi sono stati fatti per imporre regole di governance delle banche molto più severe di quelle precedentemente in uso, stabilendo anche nuovi criteri per la determinazione delle remunerazioni dei manager bancari affinché esse siano meno spropositate di prima e meno legate a incentivi ragguagliati a risultati aziendali di breve periodo.

Il fervore normativo è stato - ed è tuttora - incredibilmente forte e da regole generali si è passati a provvedimenti sempre più di dettaglio. Esso ha contraddistinto numerose autorità nazionali e internazionali, 
creando disorientamento negli ambienti bancari e finanziari e caratterizzando peraltro in modo diverso i singoli Paesi, originando situazioni concorrenziali alternativamente favorevoli o sfavorevoli alle banche sottoposte alle diverse normative.

Tale fervore ha contraddistinto anche alcune banche centrali che, resesi conto delle difficoltà che i suddetti provvedimenti hanno originato nell'effettuazione di operazioni particolarmente rischiose come i prestiti all'economia (collegati a requisiti patrimoniali sempre più stringenti che hanno finito col limitare il sostegno delle banche all'economia proprio nei momenti in cui la politica chiedeva a gran voce la ripresa) hanno inondato di liquidità il mercato. In realtà, i successi sperati di questa manovra non sono stati conseguiti e, da un lato, tale liquidità non è stata affatto impiegata per sostenere le economie e, dall'altro lato, i tassi di interesse sono clamorosamente calati scendendo addirittura sotto lo zero.

L'attuale andamento dei tassi ha penalizzato le banche, i cui spread si sono ridotti con gravi conseguenze per la loro redditività. Secondo i più recenti dati di Banca d'Italia, per rimanere a ciò che accade nel nostro Paese, nel 2014 la perversa miscela di una regolamentazione soffocante e di tassi di interesse bassissimi ha fatto sì che il ROE delle nostre banche sia stato pressoché nullo e che quello dei cinque maggiori gruppi bancari italiani sia stato addirittura negativo.

In verità, a questi risultati si è pervenuti anche perché le autorità di vigilanza, nel corso del 2014, hanno imposto alle banche una politica di bilancio molto restrittiva, costringendole a pesanti svalutazioni delle attività di bilancio e, in particolare, del portafoglio prestiti.

Alcuni pensano che il ROE suddetto possa tornare in tempi relativamente brevi ai livelli precedenti il 2007, ma - a parte il fatto che già allora esso era nettamente inferiore a quello delle banche di numerosi altri paesi e comunque non competitivo nel mercato finanziario - i dubbi in argomento rimangono elevati. La pulizia dei bilanci infatti non è ancora terminata, gli spread non danno il minimo segno di un'inversione di tendenza, il rendimento dei titoli pubblici è diventato negativo, il costo del rispetto delle regole è salito vertiginosamente, le ristrutturazioni aziendali procedono con lentezza, gli appetiti del fisco sono cresciuti e via dicendo.

Il problema è importantissimo per il futuro delle banche. Innanzitutto, se la redditività non migliora, le possibilità di aumentare il patrimonio per perseguire obiettivi di sviluppo dimensionale sono 
nulle. Non ci sarà infatti autofinanziamento e non sarà possibile reperire nuovo capitale nel mercato, in cui gli investitori troveranno opportunità migliori. Nei limiti in cui il patrimonio continuerà ad essere al centro dell'attenzione dei regolatori e dei controlli di vigilanza, questo significherà il blocco delle banche e la riduzione della loro propensione alla concessione dei crediti.

A questo specifico proposito tutti pensano peraltro che la regolamentazione subirà nuove modificazioni che potrebbero complicare ulteriormente la vita delle banche. Senza conoscere quali saranno tali modificazioni, del resto, sarà ben difficile per le stesse banche delineare le loro strategie future. Sarebbe quindi opportuno che i regolatori dichiarassero le loro intenzioni affinché le banche possano tenerle in adeguato conto.

Questi timori non sono quindi affatto teorici, ma hanno conseguenze pratiche importanti e riguardano tutta l'area dell'UE, in cui le reazioni stanno facendosi sempre più pesanti sia a livello politico sia a livello aziendale.

A livello aziendale ricordo le reazioni violente di grandi banche europee che hanno provveduto a ricambi dei vertici, a pesanti licenziamenti di personale, alla chiusura di filiali estere e di partecipate non coinvolte nel core business, a forti svalutazioni dell'attivo e così via. In questo senso, esse non fanno altro che ripetere l'esperienza che le grandi banche americane hanno fatto qualche anno fa e che si sta dimostrando vincente, al punto che la loro presenza sul mercato domestico rimane fortissima mentre quella sui mercati internazionali ha ripreso alla grande anche dopo la chiusura di una marea di filiali al di fuori degli USA.

Il sistema bancario americano non è tuttavia composto solo da grandi banche. Ve ne sono infatti circa 6.000 con un attivo di bilancio inferiore a 50 miliardi di dollari, i cui problemi sono diversi da quelli dei grandi gruppi. Ebbene, nonostante che le regole e la fiscalità siano meno pesanti che in Europa, le banche americane non grandi sono in difficoltà. I costi delle nuove regole fatte sotto la pressione della crisi e dell'obiettivo della riduzione dei rischi si stanno in effetti dimostrando insostenibili. Se questa è la loro situazione, figuriamoci la nostra.

$\mathrm{E}$, tornando proprio a quest'ultima, vorrei tentare qualche conclusione. Quanto precede dovrebbe aver dimostrato che l'eccesso di regole, l'avversione al rischio - condizionata anche dalla paura di ripetere errori che hanno così pesantemente afflitto i bilanci e le performances borsistiche $-\mathrm{e}$ il ruolo cruciale del patrimonio sono i principali fenomeni che 
mantengono la redditività delle banche a livelli bassissimi. Inoltre, essi stanno pericolosamente ridimensionando la figura delle banche come «imprese». Allorché le direttive comunitarie introdussero questo concetto nel nostro Paese, ci fu grande entusiasmo e tutti attesero notevoli benefici dalla trasformazione in imprese di banche storicamente considerate più che altro come istituzioni. Queste aspettative si basarono essenzialmente sulla speranza che le banche diventate imprese potessero servire meglio il mercato e sostenere più intensamente lo sviluppo, le imprese non finanziarie e le famiglie. Alla base di tali prospettive, implicitamente o meno, era evidente che le banche avrebbero dovuto assumere rischi maggiori. Il rischio, del resto, è una variabile connaturata alle imprese, nelle quali i proprietari apportano mezzi finanziari detti appunto capitali di rischio. È noto che, quando un'impresa (banca o non banca) è in difficoltà, i primi a risentire gli effetti negativi della crisi sono proprio i portatori del capitale di rischio, come peraltro stabilito anche dalle recenti norme sulla risoluzione delle crisi bancarie.

Per cercare di ritornare a livelli reddituali più interessanti le banche europee stanno effettuando più o meno drastiche ristrutturazioni, ma stanno anche ridimensionando i costi di produzione e di distribuzione. L'obiettivo è perseguito da un po' di tempo in qua con un'intensità sconosciuta nel passato. Gli effetti di queste azioni non sono ancora completamente visibili, ma tutto lascia sperare che qualcosa di buono stia accadendo.

Più difficile è il tentativo di rilanciare i ricavi, reso addirittura pressoché impossibile dalla situazione dei tassi di interesse e, conseguentemente, da quella degli spread. I dati di cui si dispone mostrano peraltro i primi effetti di manovre miranti a ridurre il peso relativo del margine di interesse nella formazione del reddito mediante l'aumento del peso delle commissioni. Risultati buoni sono già stati ottenuti presso le banche americane, ma anche in Italia quest'inversione di tendenza è già una realtà, che è stata associata anche ad un peggioramento dei rapporti con la clientela. Sembra infatti che l'aumento delle commissioni sia dovuto non solo allo sviluppo di nuovi e più ricchi servizi bensì anche ad addebiti poco trasparenti per operazioni non sempre richieste dai clienti e poco o niente utili alle loro imprese. Il fenomeno, negli USA, è finito sotto le potenti lenti delle associazioni dei consumatori e prima o poi accadrà qualcosa anche da noi.

Queste ultime considerazioni tirano in ballo la cultura aziendale delle banche, ferma a stereotipi antichi che stentano a modificarsi per 
tener conto dei mutamenti intervenuti nell'ambiente, nel mercato, nell'uso delle tecnologie informatiche e soprattutto nelle esigenze della clientela. In particolare, è la cultura del rischio che va cambiata. Rispettando le nuove regole imposte soprattutto negli ambiti patrimoniali e prendendo atto dell'evoluzione del mercato, le banche rischiano di non svilupparsi più e di svolgere in modo meno fattivo il ruolo di promotori e sostenitori dello sviluppo economico che è sempre stato uno dei punti fondamentali delle loro azioni.

Qui sta il perno del futuro delle banche, al quale i regolatori e le autorità di vigilanza dovrebbero prestare un'attenzione maggiore di quella prestata in questi ultimi anni. L'obiettivo della riduzione dei rischi ha portato ad una regolamentazione asfissiante, che per certi aspetti almeno momentaneamente ha avuto successo. Molte banche sono oggi meno deboli e meno rischiose di un tempo. Ma questo non è sufficiente per garantire che le crisi non ci saranno più. Tale situazione potrà infatti mantenersi solo se le regole continueranno ad essere applicate, ciò che non dipende solo dalle regole stesse bensì soprattutto dai comportamenti dalle banche nei loro confronti. Allorché esse decidessero di violare dette regole, queste non avrebbero più alcun valore e gli obiettivi perseguiti non sarebbero più raggiunti. Affinchè le regole siano efficaci occorre quindi che i comportamenti delle banche siano coerenti, ciò che non può essere assicurato da altre regole, ma solo da una cultura bancaria conforme che produca i comportamenti all'uopo necessari.

Tutto ciò non è però facile e richiede tempi non brevi e grandissima determinazione nei vertici delle banche. Se, peraltro, questi non dimostreranno di saper affrontare efficacemente la sfida del cambiamento culturale la pressione delle regole sarà destinata a crescere.

Allorchè si parla di cambiamenti culturali un riferimento indispensabile è rivolto all'etica, che può essere intesa in due modi. Il primo consiste nel ritenere che solo un'etica ispirata al bene comune può assicurare comportamenti rivolti al sostegno dello sviluppo economico e sociale per produrre un incremento del benessere collettivo. Il secondo è più limitato e più pragmatico e dà per scontato che a monte dei comportamenti ci siano decisioni aziendali che hanno previsto obiettivi adeguati anche in termini di rischio per assicurare un giusto equilibrio tra la stabilità delle banche e la soddisfazione dei loro stakeholders e, in primis, dei loro azionisti. In questi ambiti i comportamenti etici sarebbero quelli coerenti con il raggiungimento degli obiettivi prefissati.

I due concetti non sono in contrasto fra loro e possono benissimo 
coesistere. Anzi, l'ideale sarebbe proprio che essi fossero entrambi presenti nello spirito delle banche e delle risorse umane in esse impiegate.

Ho detto che cambiare cultura e comportamenti non è facile. Le difficoltà sono ancora maggiori quando si tratta di passare da una fase di attacco ad una fase di ritirata. Mi spiego. Per lunghi anni le banche hanno mirato soprattutto a sviluppare le loro attività più sotto il profilo quantitativo che sotto quello qualitativo. La crescita dimensionale è sempre stata al primo posto fra gli obiettivi delle banche, ciò che ha condizionato la loro cultura e i comportamenti delle risorse umane in esse impiegate, ma anche la politica commerciale, organizzativa e di marketing. Ora, gli anni che abbiamo di fronte avranno invece come obiettivo la riduzione dell'attività delle banche che imporrà, come del resto sta già imponendo, politiche commerciali, organizzative e di marketing profondamente diverse e, con esse, cultura e comportamenti diversi da quelli del passato, ovviamente a parità di altre condizioni, comprese quelle che hanno portato alle crisi. La «ritirata» non sarà facile e le condizioni alle quali può avere successo sono molto complesse e delicate. Le banche giocheranno qui, del resto, una partita cruciale per la loro sopravvivenza nel medio/lungo periodo. In essa dovranno avere una forte determinazione e togliersi di dosso quella paura (essenzialmente quella del rischio) che sembra caratterizzarle oggi.

In tali processi due punti saranno da tenere in conto. Il primo è rappresentato dalla qualità delle risorse umane, che stanno vivendo un momento delicato. In molti paesi, rispetto ai quali il nostro è peraltro coinvolto in misura minore, si sta infatti assistendo ad una fuga di buoni manager che, a motivo dei vincoli alla loro attività imposti dalle regole più volte citate e dalle limitazioni ai loro emolumenti imposte anch'esse da nuove regole, stanno lasciando le banche per andare a lavorare in società finanziarie che danno loro migliori prospettive di sviluppo in condizioni di maggiore libertà d'azione e con remunerazioni più elevate. Anche questo problema dovrebbe essere considerato dalle autorità politiche e bancarie per indurle a una semplificazione e a una razionalizzazione della rete di regole che sta provocando danni alla redditività delle banche rendendo problematico il loro futuro.

Quest'ultimo sarà quindi orientato ad una riduzione del peso dell'intermediazione bancaria sull'intermediazione finanziaria totale. Il fenomeno non riguarderà gli USA, dove già tale peso è molto basso e di fatto è complementare a quello dell'intermediazione svolta dai mercati finanziari e dalle shadow banks, ma riguarderà invece da vicino parte 
dei paesi europei e, in primo luogo, l'Italia, dove il rapporto citato è estremamente elevato. Dato che si ritiene che un sistema eccessivamente bancarizzato penalizzi lo sviluppo economico e, in particolare, la gestione finanziaria delle imprese e quella dei risparmiatori, le autorità governative e bancarie nazionali e internazionali stanno orientando le loro politiche verso una ritirata del mondo bancario e un potenziamento dei mercati finanziari, favorendo anche, seppure in modo implicito e indiretto, la crescita delle shadow banks. La regolamentazione va in questo senso e la dimostrazione più chiara di questa affermazione è la constatazione che il ROA delle banche in cui il rapporto fra crediti in essere e totale attivo supera il $50 \%$ è inferiore a quello delle banche in cui lo stesso rapporto è inferiore al $50 \%$, ciò che fa ritenere che l'intermediazione creditizia svolta dalle banche potrà solo ridursi, esattamente come voluto dalle suddette autorità.

Non so se questo sia un bene o un male, ma è certo che dobbiamo prendere atto che si tratta di un elemento cruciale nell'evoluzione dell'attività bancaria, che ci offrirà un mondo nuovo il quale potrà dare soddisfazione alle banche solo nei limiti in cui sapranno gestirlo a loro favore, ma che dovrà essere gestito anche nell'interesse di tutti i loro stakeholders. La sfida non è delle più semplici, ma non è affatto scontato che debba essere perduta ${ }^{1}$.

1 Chi volesse approfondire gli argomenti qui trattati può consultare il mio articolo con lo stesso titolo pubblicato sulla rivista "Strumenti finanziari e fiscalità", n. 21, dicembre 2015. 